\title{
Anesthetic Care of a Child With Acute Flaccid Myelitis
}

\author{
Anuradha Kanaparthi ${ }^{\mathrm{a}, \mathrm{b}, \mathrm{d} \text {, e }}$, Joseph D. Tobias ${ }^{\mathrm{b}, \mathrm{c}, \mathrm{d}}$
}

\begin{abstract}
Acute flaccid myelitis (AFM) is a neurological disorder that affects the anterior horn of the spinal cord, resulting in progressive weakness, loss of motor function, hypotonia and hyporeflexia. Given the progressive and frequently long-term neurological involvement of AFM, patients may require anesthetic care during radiological imaging or other surgical procedures, such as a tracheostomy or the placement of a gastrostomy tube. We present a 4-year-old girl with AFM who required anesthetic care during a tracheostomy. The end-organ involvement of the disease is discussed, pathogenesis and treatment options are reviewed and anesthetic implications are presented.
\end{abstract}

Keywords: Pediatric; Acute flaccid myelitis; Anesthesia; Neuromuscular weakness

\section{Introduction}

Acute flaccid myelitis (AFM) is a progressive neurological disorder that affects the anterior (ventral) horn of the spinal cord, resulting in lower motor neuron damage that spans multiple vertebral segments [1]. It presents with progressive weakness, loss of motor function, hypotonia and hyporeflexia [2, 3]. The Center for Disease Control and Prevention (CDC) first described AFM following a surge of cases within the United States in 2014 [4]. AFM diagnosis is suggested by the typical clinical findings, with a pleocytosis (greater than 5 white blood cells $/ \mathrm{mm}^{3}$ ) and a mild elevation of protein concentration in the cerebrospinal fluid [3]. Confirmation of the diagnosis is made by magnetic resonance (MR) imaging demonstrating characteristic destruction of the gray matter of the ventral horns of the spinal cord [5]. Antecedent respiratory and/or gastrointes-

Manuscript submitted July 29, 2019, accepted August 5, 2019

${ }^{a}$ College of Medicine, Northeast Ohio Medical University (NEOMED), Rootstown, OH, USA

${ }^{b}$ Department of Anesthesiology \& Pain Medicine, Nationwide Children's Hospital, Columbus, OH, USA

'Department of Anesthesiology \& Pain Medicine, The Ohio State University College of Medicine, Columbus, OH, USA

${ }^{\mathrm{d}}$ Authors contributed equally to this manuscript.

${ }^{\text {e}}$ Corresponding Author: Anuradha Kanaparthi, Department of Anesthesiology \& Pain Medicine, Nationwide Children's Hospital, 700 Children's Drive, Columbus, OH 43205, USA. Email: akanaparthi@neomed.edu

doi: https://doi.org/10.14740/jmc3337 tinal viral illness with or without fever occurs in the majority of AFM cases $[1,6,7]$. The proximal muscle groups of the upper extremities are more commonly affected, typically with a relative preservation of distal limb motor function. Cranial nerve involvement with bulbar dysfunction may also occur, as AFM may affect gray matter of the brainstem (particularly, the pons), resulting in diplopia and facial muscle weakness due to affected cranial nerves VI and VII $[3,7]$. Given the progressive and frequently long-term neurological involvement of AFM, patients may require anesthetic care during radiological imaging or other surgical procedures. We present a 4-year-old girl with AFM who required anesthetic care during a tracheostomy and percutaneous endoscopic gastrostomy (PEG) placement. The end-organ involvement of the disease is discussed, pathogenesis and treatment options are reviewed and anesthetic implications are presented.

\section{Case Report}

Institutional Review Board approval is not required for single case reports at Nationwide Children's Hospital (Columbus, $\mathrm{OH})$. The patient was a previously healthy 4 -year-old, $21.5 \mathrm{~kg}$ girl presenting for a PEG tube placement and tracheostomy secondary to respiratory failure due to neuromuscular weakness. She presented initially to the emergency department (ED) 3 weeks prior to the date of current surgery for complaints of bilateral weakness of the upper and lower limbs (inability to stand), flaccid paralysis, areflexia, hypotonia and bulbar dysfunction. Her past medical history included a viral upper respiratory illness with cough, rhinorrhea and congestion for 2 weeks prior to admission. A diagnosis of AFM was made following MR imaging, which demonstrated bilateral destruction of the gray matter, largely restricted within the ventral horns of the cervical and thoracic spinal cords. Lumbar puncture was performed, and the cerebrospinal fluid showed a normal protein and glucose with 1 red blood cell and 3 white blood cells per $\mathrm{mm}^{3}$. The patient was treated with intravenous immunoglobulin (total dose of $2 \mathrm{~g} / \mathrm{kg}$ ) with minimal improvement in strength. Due to ongoing motor weakness, she required mechanical ventilation, and a direct laryngoscopy with placement of a tracheostomy and laparoscopic gastrostomy tube (PEG) was planned. Previous surgical procedures had been delayed twice due to preoperative hemodynamic instability with hypotension and intraoperative hypoxemia after induction of anesthesia of unclear etiology. A gastrostomy was initially scheduled approximately 1 week after admission to ED; however, it was cancelled due to progressive respiratory failure with worsening oxygenation and atelectasis on the morning of the 
procedure. After stabilization, the second planned operative procedure included tracheostomy and PEG placement. However, upon transferring the patient to the operating room table, decreased oxygen saturation and absent breath sounds on the left side were noted. A chest radiograph performed revealed left-sided atelectasis. After removal of a large mucous plug via rigid bronchoscopy, ventilation and oxygenation improved. However, the planned procedure was cancelled, the patient was transferred to the pediatric intensive care unit (PICU) and the procedure was rescheduled for the following week. On the morning of the procedure 1 week later, the patient's respiratory status was stable with a low oxygen requirement and no evidence of atelectasis on the morning chest radiograph. The patient was kept nil per os after midnight and intravenous fluids were started. Anesthetic plan, risks, benefits and alternatives were discussed with the parents and informed consent was obtained. The patient was transported to the operating room from the PICU. American Society of Anesthesiologists (ASA) monitors were applied. Anesthesia was induced with propofol and fentanyl. Neuromuscular blockade was provided by rocuronium. Infusions of midazolam and hydromorphone, which were being administered in the PICU, were continued during the case. Additional maintenance anesthesia included sevoflurane in air/oxygen. She was placed in a supine position, with arms tucked to the side. A second peripheral intravenous cannula was placed. Intraoperatively during the procedure, there were intermittent episodes of hypotension (blood pressure $50 / 33 \mathrm{~mm} \mathrm{Hg}$ ) which were treated with bolus doses of phenylephrine. During these episodes of hypotension, there were associated decreases in oxygen saturation (pulse oximeter reading as low as $30 \%$ ). During the procedure, a total of five bolus doses of phenylephrine $(1-2 \mu \mathrm{g} / \mathrm{kg})$ were administered. Both the blood pressure and oxygen saturation returned to normal with the intermittent phenylephrine doses. The blood pressure instability was assumed to be due to autonomic dysfunction related to AFM. The procedure lasted approximately $2.5 \mathrm{~h}$ during which time a PEG tube and tracheostomy were placed. Intravenous fluids included $165 \mathrm{~mL}$ of maintenance fluids, $5 \%$ dextrose in $1 / 2$ normal saline and $160 \mathrm{~mL}$ of lactated Ringers. The patient was transferred to the PICU, and the remainder of her postoperative course was unremarkable. Over the next 3 4 weeks, the patient's need for mechanical ventilation support was weaned. The tracheostomy and PEG tube were removed successfully approximately 2 months after placement. Her current status includes residual weakness and spasticity which impair activities of daily life and mobility. Specifically, she has residual weakness in her arms and legs with spastic tetraplegia, the right side being more affected than her left. She continues to participate in physical therapy and rehabilitation.

\section{Discussion}

AFM typically starts with a prodromal febrile viral illness of the upper respiratory or gastrointestinal tracts $[1,6]$. Symptoms are typical of viral infections including cough, rhinorrhea, and congestion or vomiting and diarrhea, generally lasting approximately 1 week. Following this, an acute neurological phase with flaccid muscle weakness, as described above, is noted [3]. Although there is generally recovery of motor function over time, residual weakness and paralysis is frequent and, at times, irreversible. Fatemi et al noted that only $5 \%$ of AFM cases in 2014 showed full recovery to previous strength [7]. Treatment of AFM consists primarily of supportive care, including endotracheal intubation with mechanical ventilation and provision of adequate nutrition. Various treatments targeting the immune system have been offered with limited success, including plasmapheresis, intravenous immunoglobulin and corticosteroids $[3,7,8]$. Anecdotal reports outline the use of antiviral agents, such as fluoxetine, which may counteract EVD68 $[7,9]$.

The specific etiology of AFM remains unclear. However, there are several enteroviruses that have been implicated in the pathogenesis, the most common being EV-D68 and EVA71 [7]. Herpes viruses, adenoviruses and flaviviruses, such as West Nile and Japanese encephalitis viruses, have also been implicated, although less frequently [7]. Despite this association with viral infections, causality has not been established between any specific virus and AFM. Given the overlap in clinical signs and symptoms, AFM can be mistaken for Guillain-Barre syndrome (GBS), transverse myelitis and poliomyelitis [5]. Definitive diagnosis requires MR image that demonstrates bilateral destruction of gray matter that is limited to the ventral horns of the spinal cord. MR imaging of GBS typically demonstrates enhancement of anterior nerve roots in the conus medullaris and cauda equina [10]. Sensory symptoms may be more indicative of transverse myelitis [11]. The absence of poliovirus in stool should be confirmed to exclude poliomyelitis as the diagnosis [3].

As with any anesthetic care, appropriate preoperative preparation begins with a thorough preoperative examination and identification of end-organ effects of the disease process. In patients with AFM, primary anesthetic concerns include the potential for involvement of the upper airway and the cardiovascular and respiratory systems. Bulbar weakness and poor upper airway control may predispose these patients to aspiration. Based on the patient's central nervous system involvement and nil per os status, techniques to limit aspiration during anesthetic induction, including rapid sequence intubation and airway control with endotracheal intubation, may be indicated $[12,13]$. As our patient's airway had been previously secure with endotracheal intubation, these issues were already addressed.

Our patient had previously developed preoperative mucus plugging, atelectasis and hypoxemia necessitating cancellation of the scheduled procedure. These issues are also relevant during the perioperative period when the residual effects of anesthetic agents and neuromuscular blocking agents may exacerbate poor baseline respiratory function and result in perioperative respiratory insufficiency. General precautions include postoperative monitoring of respiratory function in an ICU setting, tracheal extubation when patient is fully awake following dissipation of residual effects of intraoperative anesthetic agents, complete reversal of residual neuromuscular blockade and judicious use of opioid analgesia. Extubation to non-invasive techniques of respiratory support such as bilevel positive airway pressure with a slow transition to unassisted breathing 
may facilitate postoperative tracheal extubation and prevent atelectasis $[14,15]$. Frequent chest physiotherapy, including assisted cough devices and incentive spirometry, may also help to facilitate the clearance of mucus and prevent postoperative respiratory complications.

The central and peripheral nervous system involvement of AFM may further impact the perioperative choice of neuromuscular blocking agents. Although not required for all surgical procedures, depolarizing and non-depolarizing neuromuscular blocking agents raise concerns regarding safety and effective blockade reversal, respectively. Due to denervation of the neuromuscular junction by succinylcholine, a depolarizing muscle relaxant, proliferation of extrajunctional acetylcholine receptors can be expected with the potential for an exaggerated hyperkalemic response. Although there are no reports regarding the administration of succinylcholine to a patient with AFM, hyperkalemia has been reported following the administration of succinylcholine to patients with GBS $[16,17]$. The similar involvement seen with AFM and GBS would suggest that succinylcholine should be avoided in patients with AFM. As our patient would receive postoperative mechanical ventilation, neuromuscular blockade was established with rocuronium, a non-depolarizing muscle relaxant. With pre-existing hypotonia and weakness, the effects of rocuronium can be expected to be prolonged; however, the novel agent, sugammadex, offers an option for rapid reversal of neuromuscular blockade with rocuronium and a limited chance of residual weakness even in patients with comorbid neuromuscular conditions [18, 19].

A final issue noted in our patient was the potential for autonomic instability and intraoperative hypotension due to spinal cord involvement, similar to spinal shock with traumatic injury [20]. Hypotension was noted, which we would postulate is related to decreased systemic vascular resistance (SVR) with bradycardia and limited tachycardic response to blood pressure changes. These cardiovascular effects may be exaggerated by the hemodynamic effects of the anesthetic agents. Treatment options include the administration of fluids and the treatment of low SVR with the direct-acting $\alpha$-adrenergic agonist phenylephrine.

In summary, we present the perioperative care of a child with AFM. Primary anesthetic concerns include the potential for involvement of the upper airway and respiratory systems, with hypotonia, poor airway control and potential for postoperative respiratory failure. Central and peripheral nervous system involvement impacts the perioperative use of neuromuscular blocking agents, while hemodynamic instability may be seen related to spinal cord involvement.

\section{Acknowledgments}

Not applicable.

\section{Financial Disclosure}

None to declare.

\section{Conflict of Interest}

None to declare.

\section{Informed Consent}

Obtained.

\section{Author Contributions}

Anuradha Kanaparthi: review of the medical record with preparation of the case report and the manuscript (initial and revised versions). Joseph D. Tobias: financial support for the project, conceptualizing the design of the report, and critical revision of all versions of the manuscript.

\section{References}

1. Donahue ML, Clausen SS, Malloy A, Dennison DH, Falcon J. Acute flaccid myelitis: Case report. MSMR. 2019;26(1):17-19.

2. Beal JA. Acute Flaccid Myelitis: A rare but devastating illness. MCN Am J Matern Child Nurs. 2019;44(2):116.

3. Messacar K, Schreiner TL, Van Haren K, Yang M, Glaser CA, Tyler KL, Dominguez SR. Acute flaccid myelitis: A clinical review of US cases 2012-2015. Ann Neurol. 2016;80(3):326-338.

4. Burton A. Outbreaks of acute flaccid myelitis? What neurologists need to know. Lancet Neurol. 2019;18(3):234235.

5. The United Kingdom Acute Flaccid Paralysis Afp Task F. An increase in reports of acute flaccid paralysis (AFP) in the United Kingdom, 1 January 2018-21 January 2019: early findings. Euro Surveill. 2019;24(6).

6. Stelzer-Braid S, Rawlinson W. Outbreaks of acute flaccid myelitis in the US. BMJ. 2018;363:k5246.

7. Fatemi Y, Chakraborty R. Acute flaccid myelitis: a clinical overview for 2019. Mayo Clin Proc. 2019;94(5):875881.

8. Kane MS, Sonne C, Zhu S, Malhotra A, Van Haren K, Messacar K, Glaser CA. Incidence, risk factors and outcomes among children with acute flaccid myelitis: a populationbased cohort study in a California health network between 2011 and 2016. Pediatr Infect Dis J. 2019;38(7):667-672.

9. Messacar K, Sillau S, Hopkins SE, Otten C, Wilson-Murphy M, Wong B, Santoro JD, et al. Safety, tolerability, and efficacy of fluoxetine as an antiviral for acute flaccid myelitis. Neurology. 2019;92(18):e2118-e2126.

10. Alkan O, Yildirim T, Tokmak N, Tan M. Spinal MRI findings of guillain-barre syndrome. J Radiol Case Rep. 2009;3(3):25-28.

11. Wang C, Greenberg B. Clinical approach to pediatric transverse myelitis, neuromyelitis Optica spectrum disorder and acute flaccid myelitis. Children (Basel). 2019;6(5). 
12. Tobias JD. Rapid sequence intubation: What does it mean? Does it really matter? Saudi J Anaesth. 2014;8(2):153154.

13. Benington S, Severn A. Preventing aspiration and regurgitation. Anaesth Intensive Care Medicine. 2007;8:368372.

14. Jaber S, Michelet P, Chanques G. Role of non-invasive ventilation (NIV) in the perioperative period. Best Pract Res Clin Anaesthesiol. 2010;24(2):253-265.

15. Pelosi P, Jaber S. Non-invasive respiratory support in the perioperative period. Curr Opin Anaesthesiol. 2010;23:2233-2228.

16. Feldman JM. Cardiac arrest after succinylcholine administration in a pregnant patient recovered from Guillain-
Barre syndrome. Anesthesiology. 1990;72(5):942-944.

17. Dalman JA, Verhagen WI. Cardiac arrest in GuillainBarre and the use of suxamethonium. Acta Neurol Belg. 1994;94:259-261.

18. Tobias JD. Current evidence for the use of sugammadex in children. Paediatr Anaesth. 2017;27(2):118-125.

19. Tezcan B, Bolukbasi D, Kazanci D, Turan S, Suer Kaya G, Ozgok A. The use of sugammadex in a patient with Guillain-Barre syndrome: a case report. A A Case Rep. 2017;8(8):200-202.

20. Bilello JF, Davis JW, Cunningham MA, Groom TF, Lemaster D, Sue LP. Cervical spinal cord injury and the need for cardiovascular intervention. Arch Surg. 2003;138(10):1127-1129. 